\title{
1st Biennial Meeting of the Eurasian Colorectal Technologies Association (ECTA), 13-15 November 2009, Guangzhou/Canton, China (Correction)
}

\author{
(C) Springer-Verlag 2010
}

Tech Coloproctol (2010) 14:57-80

DOI 10.1007/s10151-010-0565-4

Unfortunately, two abstracts were missing in the published abstracts. Therefore, please find the two abstracts here.

\section{Long-term outcome of loose-Seton for complex anal fistulas in patients with and without Cohn's disease-a multi-center study \\ Y. Ziv, E. Galis-Rozen, A. Stepanski, A. Rosen, S. Eldar, M. Rabau, H. Tulchinsky \\ Department of Surgery, Tel Aviv Sourasky Medical Center, Asaf-Harofeh Medical Center, Zerifin, Israel}

Background Conflicting results to treat complex anal fistulas have been reported. We analyse the results of permanent loose-Seton in the management of high anal fistulas in patients with Crohn's disease (CD) and two-stage Seton fistulotomy in patients without CD. Methods A retrospective study (2004-2008) of all patients treated with loose-Seton for complex anal fistula treate. Data on demographics, medical history, type of fistula, disease duration, prior surgeries, morbidity, recurrence and mortality, were collected. Results 60 patients without CD underwent 107 fistula-related surgical procedures, and $17 \mathrm{CD}$ patients underwent 29 procedures. Early post operative complications, mainly local sepsis or bleeding were recorded in $8(10 \%)$ patients. Long term complications were observed in $9(15 \%)$ control patients and $4(23 \%)$ CD patients. During a median follow-up period of 24 months, recurrence rate was $40 \%$ in CD patients and $47 \%$ in patients without $\mathrm{CD}$. Five of $77(6 \%)$ patients $(4$ non $\mathrm{CD}$ patients, one $\mathrm{CD}$ patient) developed some degree of faecal incontinence. Conclusion Two-stage Seton fistulotomy is associated with high recurrence rate. Other interventions, such as advancement flap repair, should be considered in some non-CD patients with complex anal fistulas. In CD patients the use of permanent loose-Seton is effective in controlling local sepsis and has low rates of subsequent incontinence.

The abstracts of the meeting can be found at doi:10.1007/s10151-010-0565-4.

\author{
Anastomotic Leaks in Rectal Cancer-An Update \\ Y. Ziv \\ Division of Surgery, Assaf-Harofe Medical Center, \\ Tel-Aviv University, Zerifin, Israel
}

Anastomotic leak is one of the most frightening surgical complications following rectal resection. Anastomotic leak has significant consequences and is associated with a $5-40 \%$ mortality rate, and a $10-100 \%$ risk of permanent stoma. Anastomotic leaks in patients with rectal cancer affect long-term outcome, functional results and decreased long-term survival due to a greater prevalence of loco regional recurrence. The incidence of rectal anastomotic leaks varies widely, (1-35\%) depending on; anastomotic type, distance of anastomotic line from the anal verge, tumor stage, gender, co-morbidities, and whether the surgery was performed emergently or electively, and whether large or small volumes of operation are performed by the surgeon. Decreased incidence of anastomotic leaks was found with the introduction of circular stapler and when surgery is performed by experienced surgeons (Colorectal vs. General Surgeons).

Identification of risk factors for anastomotic leakage may lead to preventive measures like controlling co-morbidities (diabetes, steroids etc.), gentle handling of large tumors (decrease perforation rate and tumor spread). Forms of anastomoses (straight colo-anal, Jcolonic pouch, coloplasty) and operative techniques (open vs. laparoscopy, notes and robotics) are compared. Should we always or selectively use de-functioning stomas for sphincter saving procedures?, what type of stoma is preferred (ileostomy, colostomy, loop, and end)?, Should we use different methods of anastomotic line re-enforcement?, Should we use intra-luminal stent?- those questions are not settled yet.

Timing of diagnosis of anastomotic leak is critical and suspicion should always be in every surgeons mind. Treatment of rectal anastomotic leaks is different in patients who present with free perforation and associated peritonitis and those with contained leaks. Free perforations are treated with explorative laparotomy, takedown of the anastomosis and creation of an end stoma, or a proximal diversion via a loop ileostomy. Contained leaks are treated conservatively, with percutaneous or trans anal canal drainage. Experimental and clinical studies on anastomotic leaks in patients with rectal cancer are reviewed. 